\title{
BMJ Open Telehealth versus self-directed lifestyle intervention to promote healthy blood pressure: a protocol for a randomised controlled trial
}

\author{
Mohamed Taher, ${ }^{1}$ Christina Yule, ${ }^{1}$ Heather Bonaparte, ${ }^{1}$ Sara Kwiecien, ${ }^{1}$ \\ Charlotte Collins, ${ }^{2}$ Allison Naylor, ${ }^{3}$ S P Juraschek, ${ }^{4}$ Lisa Bailey-Davis (1) , ${ }^{3,5}$ \\ Alex R Chang (iD) ${ }^{1,5}$
}

To cite: Taher M, Yule C Bonaparte $\mathrm{H}$, et al. Telehealth versus self-directed lifestyle intervention to promote healthy blood pressure: a protocol for a randomised controlled trial. BMJ Open 2021;11:e044292. doi:10.1136/ bmjopen-2020-044292

- Prepublication history for this paper is available online. To view these files, please visit the journal online (http://dx.doi org/10.1136/bmjopen-2020044292).

Received 31 August 2020 Revised 27 November 2020 Accepted 18 February 2021

Check for updates

(C) Author(s) (or their employer(s)) 2021. Re-use permitted under CC BY-NC. No commercial re-use. See rights and permissions. Published by BMJ.

${ }^{1}$ Kidney Health Research Institute, Geisinger, Danville, Pennsylvania, USA

${ }^{2}$ Center for Professionalism, Geisinger, Danville, Pennsylvania, USA

${ }^{3}$ Obesity Institute, Geisinger, Danville, Pennsylvania, USA ${ }^{4}$ Medicine, Beth Israel Deaconess Medical Center, Boston, Massachusetts, USA ${ }^{5}$ Department of Population Health Sciences, Geisinger, Danville, Pennsylvania, USA

Correspondence to

Dr Alex R Chang;

achang@geisinger.edu

\section{ABSTRACT}

Introduction Weight loss, consumption of a Dietary Approaches to Stop Hypertension dietary pattern, reduced sodium intake and increased physical activity have been shown to lower blood pressure (BP). Use of web-based tools and telehealth to deliver lifestyle counselling could be potentially scalable solutions to improve BP through behavioural modification though limited data exists to support these approaches in clinical practice.

Methods and analysis This randomised controlled trial will compare the efficacy of a telehealth versus selfdirected lifestyle intervention in lowering 24-hour SBP in patients with overweight/obesity (body mass index $\geq 25$ $\mathrm{kg} / \mathrm{m}^{2}$ ) and 24-hour SBP 120-160 mm Hg. All participants receive personalised recommendations to improve dietary quality based on a web-based Food Frequency Questionnaire, access to an online comprehensive weight management programme and a smartphone dietary app. The telehealth arm additionally includes weekly calls with registered dietitian nutritionists who use motivational interviewing. The primary outcome is change from baseline to 12 weeks in 24-hour SBP. Secondary outcomes include changes from baseline in 24-hour diastolic BP, daytime SBP, nighttime SP, daytime diastolic BP, nighttime diastolic BP, total Healthy Eating Index-2015 score, weight, waist circumference and physical activity. Other prespecified outcomes will include change in individual components of the Healthy Eating Index-2015 score, and satisfaction with the Healthy BP research study measured on a 5-point Likert scale.

Ethics and dissemination The study has been approved by the Geisinger Institutional Review Board. Results will be disseminated through peer-reviewed publications and conference presentations.

Trial registration number NCT03700710.

\section{INTRODUCTION}

The American College of Cardiology/American Heart Association (ACC/AHA) 2017 Hypertension Guidelines redefined hypertension as having two blood pressure (BP) readings measured on at least two separate occasions $\geq 130 / 80 \mathrm{~mm} \mathrm{Hg} .{ }^{1}$ With this updated

\section{Strengths and limitations of this study}

- This randomised controlled trial will compare the efficacy of a telehealth versus self-directed lifestyle intervention in lowering blood pressure through lifestyle modification in patients with elevated blood pressure and overweight/obesity.

- The proposed interventions under investigation are low-cost and potentially scalable.

- Primary end point data will be collected using 24-hour ambulatory blood pressure monitoring at baseline and at 12 weeks, and additional secondary endpoint data will be collected including Healthy Eating Index-2015 score assessed by Food Frequency Questionnaire, weight, waist circumference and physical activity, assessed by questionnaire.

- While participants and some of the study staff are unable to be blinded, researchers assessing study outcomes and conducting analyses will be blinded to the arms.

- A limitation is that there is no control arm receiving no intervention at all.

definition, nearly half of the US adult population has hypertension. ${ }^{2}$ Obesity, Western-style dietary patterns rich in sodium and processed foods, and low physical activity have been implicated in the pathogenesis of hypertension and cardiovascular diseases. ${ }^{13}$ The ACC $/{ }^{1}$ AHA Hypertension guidelines recommend lifestyle modification as class I recommendations for all patients with hypertension as well as those with elevated systolic BP (SBP) (120-129 mm Hg) since weight reduction, consumption of a low-sodium Dietary Approaches to Stop Hypertension (DASH)type diet, and increased physical activity can lower BP substantially. ${ }^{4-6}$

Despite recognition that addressing lifestyle behaviours is of high importance to address cardiometabolic risk, delivery of lifestyle modification in clinical care remains 
challenging due to time constraints, lack of referral resources and other competing priorities. ${ }^{78}$ Some possibilities to address this gap include the use of app-based interventions and the use of telehealth to deliver lifestyle counselling remotely. A recent systematic review and meta-analysis found that app-based interventions have the potential for aiding patients make healthy nutrition behaviour changes and improving nutrition-related health outcomes. ${ }^{9}$ Prior studies have found that telehealth lifestyle coaching interventions are similarly effective as traditional, in-person delivered interventions for helping patients achieve weight loss. ${ }^{10}{ }^{11}$ However, limited data exist on the efficacy of telehealth-delivered strategies in lowering BP through lifestyle modification. Most BP telehealth studies typically include remote monitoring of BP and antihypertensive medication, making it difficult to assess the impact of lifestyle modification. ${ }^{12} 13$

It remains unclear whether telehealth lifestyle counselling further lowers BP through healthy behaviour modification on top of app-based interventions. To help inform health systems and payers in devising scalable, low-cost strategies to improve hypertension control through lifestyle modification, we aim to compare the efficacy of a self-guided versus a telehealth dietitian-led approach using web-based tools in reducing 24-hour SBP from baseline to 12 weeks.

\section{METHODS AND ANALYSIS \\ Study design/setting}

The Healthy BP study is a randomised controlled trial (RCT) comparing a self-guided versus a dietitian-led telehealth approach using web-based tools to help patients lower BP from baseline to 12 weeks through lifestyle modification. The study intervention period of 12 weeks was chosen as prior studies suggest this time frame is adequate to observe an improvement in BP with lifestyle modification $^{31415}$ and to minimise the chance that a patient in either arm would undergo a change in antihypertensive medication. The RCT is being conducted at two sites (Danville, Pennsylvania, USA Wilkes-Barre, Pennsylvania, USA) within the Geisinger Health System. Recruitment began 16 January 2019.

\section{Study population}

A total of 200 participants will be included. Eligibility criteria include $\geq 18$ years of age, body mass index (BMI) $>25 \mathrm{~kg} / \mathrm{m}^{2}$, access to a telephone and either a computer or smartphone with internet access, 24-hour ambulatory SBP between 120-160 mm Hg, and successful completion of the run-in period (enter dietary data entry into LoseIt app (www.loseit.com) for at least 5 out of 7 days; enter weight into a comprehensive weight management website (www.bmiq.com)). Exclusion criteria include: inability to understand English, myocardial infarction, stroke or atherosclerotic cardiovascular disease within prior 6 months, planned or previous bariatric surgery, pregnancy, breast feeding or planned pregnancy prior to the end of participation, self-reported average consumption of $>21$ alcoholic beverages per week or binge drinking, psychiatric hospitalisation in past year, current angina or plans to leave the area prior to the end of the study, current participation in another clinical trial, and principal investigator discretion (ie, concerns about safety, compliance). The broad 24-hour SBP inclusion range was chosen to allow participation among patients recommended to make lifestyle modifications per the ACC $/{ }^{1} \mathrm{AHA}$ Hypertension Guidelines. ${ }^{1}$ For patients with 24-hour or daytime $\mathrm{SBP} \geq 145 \mathrm{~mm} \mathrm{Hg}$, the principal investigator discussed the results with patients and their providers on whether they wished to start or escalate antihypertensive medication therapy, or commit to making lifestyle modifications through the research trial. Patients who had antihypertensive medication started or escalated could be re-evaluated for eligibility with 24-hour ambulatory BP monitoring (ABPM) at least 2 weeks after the medication change.

\section{Interventions}

All participants in both arms will receive instructions to access the BMIQ website and set up an account on LoseIt, a meal-logging app that integrates with the BMIQ website. BMIQ is an evidence based, Health Insurance Portability and Accountability Act (HIPAA)-compliant programme developed by Louis Aronne. ${ }^{16}$ Educational materials on the BMIQ website include materials to promote weight loss, eating a DASH-type diet (increasing intake of fruits/ vegetables, whole grains, protein sources from plants, lean meats, and seafood, and decrease sodium, sugar intake and saturated fat), reducing sodium intake, increasing physical activity, setting goals, overcoming barriers and relapse prevention. A list of the session topics, with a few customisations by the study team, are shown in table 1 . Participants also will receive a personalised nutrition report based on the Viocare Food Frequency Questionnaire (FFQ) that suggest ways to improve their dietary habits to best align with national dietary guidelines. ${ }^{17}$ If participants encounter problems with using LoseIt or BMIQ, research staff will provide assistance. If no data are logged for more than a week, research staff will reach out to patients via text messages or phone calls to help troubleshoot any problems. To encourage adherence with dietary data entry, participants will receive virtual lottery tickets (lottery drawings for 10 US $\$ 300$ gift certificates) each week in which they enter at least 5 days/week of dietary data and 2 days/week of weights. All participants will be offered the opportunity to participate in grocery store tours at a local grocery store (Weis Markets).

Overarching participant goals include: (1) weight loss of $3 \%$ at 12 weeks; (2) consume a healthier dietary pattern (high in fruits, vegetables, whole grains, low-fat dairy, vegetable/fish/poultry sources of protein, healthier sources of fat and avoid sugar and salt); (3) reduce sodium intake to $<2300 \mathrm{mg} /$ day and (4) at least $180 \mathrm{~min} /$ week of moderate/vigorous intensity physical activity.

The self-guided intervention group will receive access to web materials but no additional support from dietitians. 


\begin{tabular}{ll}
\hline Table 1 & BMIQ session topics \\
\hline Week 1 & $\begin{array}{l}\text { Welcome! getting started, self-monitoring } \\
\text { diet and sodium }\end{array}$ \\
\hline Week 2 & Healthy eating for weight loss \\
Week 3 & Walk off the weight \\
\hline Week 4 & Setting SMART goals for weight control \\
Week 5 & Gaining control of your food environment \\
\hline Week 6 & Take the bite out of stress eating \\
\hline Week 7 & Social cues \& you \\
\hline Week 8 & Pump up my activity plan \\
\hline Week 9 & Navigating restaurants \& grocery stores \\
\hline Week 10 & Don't let slips trip you up! \\
\hline Week 11 & Feeding your mind \\
\hline Week 12 & Sharpening my problem-solving skills \\
\hline Week 13 & Don't let success slip away \\
\hline Week 14 & Graduation! \\
\hline (Optional) & Do it yourself meals \\
\hline (Optional) & Food cravings \\
\hline (Optional) & Getting a hold over hunger \\
\hline (Optional) & How successful weight losers do it \\
\hline (Optional) & Incorporating highly desired foods \\
\hline (Optional) & Navigating the food label \\
\hline Optional) & Triple trouble: sugar, fat and salt \\
\hline & \\
\hline
\end{tabular}

The telehealth dietitian-led intervention group additionally will receive weekly telephone calls (initial call 30-60 min, follow-up calls 15-20 min) from a study dietitian. This approach is informed by Carver and Scheier's control systems theory ${ }^{18}$ and the situated learning theoretical framework. ${ }^{19}$ Control systems theory describes human behaviour as a 'continual process of moving toward, and away from, various kinds of mental goal representations, and this movement occurs by a process of feedback control.' By using the app and website and conversing with dietitians remotely, situated learning will occur through activities and experiences in the context of participants' own environments. Dietitians in this study explore participants' goals and values and help patients set an attainable goal around a health behaviour for the week and assess their progress towards those goals using motivational interviewing techniques and principles from. Motivational interviewing skills will be continually developed through biannual training sessions by a motivational interviewing expert. Dietary data entered in the LoseIt app is used to estimate average daily intake of sodium, fruits and non-starchy vegetables, which can be used for guidance for goal-setting around sodium intake and fruit and vegetable intake.

\section{Recruitment and baseline 24-hour ABPM}

Participants will be recruited using multiple methods from 16 January 2019 to approximately 28 February 2021 (7-8 per month). The primary source of recruitment will
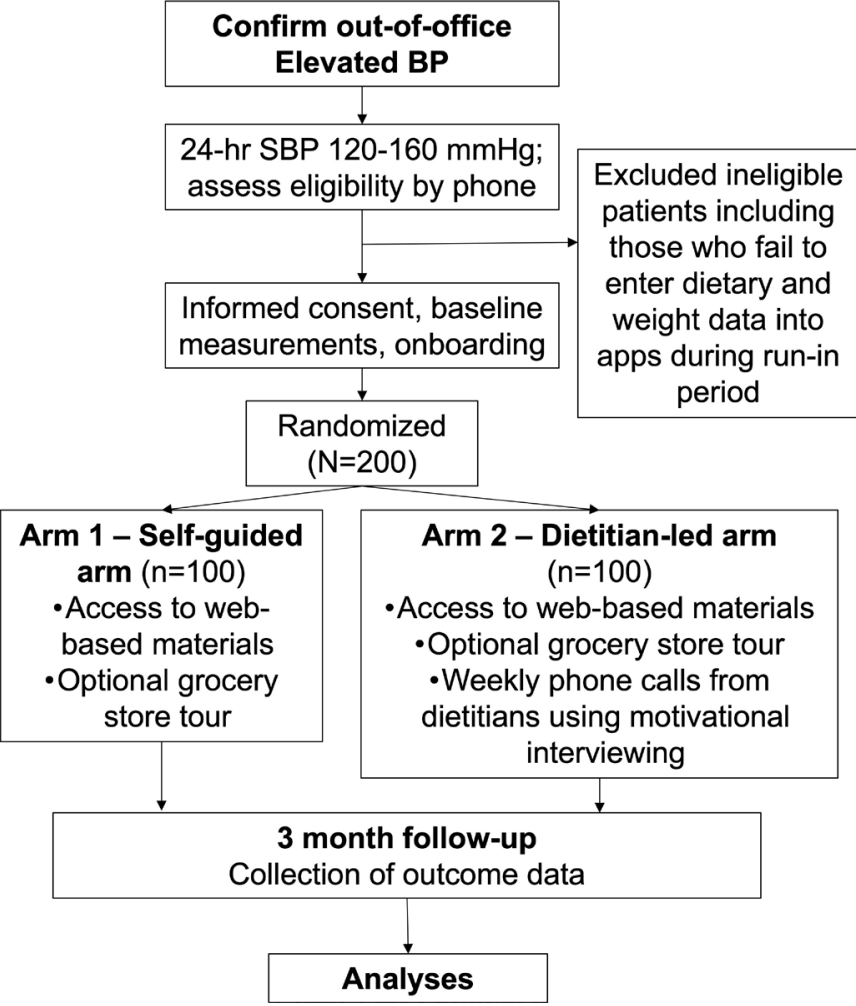

Figure 1 Study flow. BP, blood pressure; SBP, systolic blood pressure.

be done by identifying patients with elevated outpatient BP using Geisinger electronic health record data. Additional sources of recruitment will include patients with Geisinger Health Plan insurance who have elevated BP during health screenings and referrals from Geisinger providers and self-referrals. Potential participants will then be invited to complete a baseline 24-hour ABPM to confirm their elevated BP outside the office as recommended by guidelines. ${ }^{20}$ The 24 hours ABPM will be provided to participants free of charge, using SpaceLabs Ontrak devices. Participants will be asked to retake the 24-hour ABPM if fewer than 14 daytime measurements or 7 night-time measurements are obtained. Awake and sleep times will be self-reported; if participants fail to report their awake and sleep times on the activity diary, the SpaceLabs Ontrak activity monitor will be examined visually to determine awake and sleep times. ${ }^{21}$ The study flow is shown in figure 1 .

\section{Baseline study visit, Run-in period, randomisation and blinding}

Participants who meet inclusion criteria are then invited to an initial study visit where informed consent will be obtained, along with weight, waist circumference and completion of Viocare FFQ ${ }^{17}$ the International Physical Activity Questionnaire (IPAQ) Short Form ${ }^{22}$ and other study questionnaires.

Participants will register for a BMIQ web account, download the LoseIt app, and shown how to sync LoseIt to BMIQ and access various features of LoseIt and BMIQ. 
Participants will then be instructed to enter dietary data daily and weight data during the run-in period and throughout the trial. If participants enter complete dietary data (three eating occasions per day) for $5 / 7$ days and enter their weight into the BMIQ website, they will be eligible to continue to randomisation.

Participants who are struggling with completing dietary data entry during the run-in period will be contacted by telephone to inquire about any issues, and the run-in period will be extended until at least 5 out of 7 consecutive days of dietary data entry is complete. Participants who complete the run-in period will then be contacted by telephone for randomisation assignment. Randomisation assignments will be generated by a computer program, and an unblinded staff member will open a sealed opaque envelope with the randomised intervention sequence, and notify participants of their intervention group assignment.

Participants, dietitians and the member of the study team assigning randomisation cannot be blinded. All other members of the study team are blinded, including study team members assessing outcomes and conducting statistical analyses.

\section{Outcome assessment}

Outcomes will be assessed as the mean (continuous) change from baseline to the 12-week follow-up visit. The primary outcome is change in 24-hour SBP, assessed using ABPM with SpaceLabs Ontrak ABPM devices. ${ }^{21}$ Secondary outcomes include changes in 24-hour diastolic BP (DBP), daytime SBP, night-time SBP, daytime DBP, night-time DBP, total Healthy Eating Index (HEI)-2015 score, weight, waist circumference, 24-hour DBP, clinic SBP, clinic DBP and physical activity (total metabolic equivalent of task (MET)-min per week). The HEI-2015 score will be assessed using the Viocare FFQ ${ }^{23}$ and total MET-min per week assessed using the IPAQ-short form. Weight will be measured without shoes or outerwear using a calibrated scale in clinic or by home scales-in the case of remote research visits necessitated by the Novel COVID-19 pandemic (table 2). Other outcomes include change in individual components of the HEI-2015 score and patient satisfaction (5-point Likert score).

\section{Adaptations to trial during COVID-19 pandemic}

In late March 2020 and onwards, adaptations were made due to the COVID-19 pandemic, which necessitated minimising in-person contacts to preserve clinic space to allow for social distancing and conserving personal protective equipment (table 2). While we were able to continue primary outcome data collection with 24-hour ABPM, the requisite shift to primarily remote study visits required us to instruct patients to self-measure weight and waist circumference, which could potentially result in some underestimation. ${ }^{24}{ }^{25}$ As we are unable to perform automated office BP in most participants during the pandemic, change in automated office BP measures were removed as a secondary outcome. Fortunately, we were
Table 2 Adaptations to trial during COVID-19

Research personnel unable to conduct inperson visits from 16 March 2020-current.

Recruitment Recruitment reliant on referrals.

Baseline 24 Increased collaboration with clinical staff to hours ABPM perform 24 hours ABPM testing for patients with elevated outpatient blood pressure readings

Baseline Study visits planned for late March 2020 were study visit postponed until remote adaptations were made and approved by the IRB. Remote baseline study visits began in May 2020 using telephone calls and study materials mailed or dropped off at participants' houses. Weight and waist circumference measured using home scales and tape measures (rather than in clinic); automated office blood pressure unable to be performed.

$\begin{array}{ll}\text { Grocery store Weis Markets introduced a virtual grocery } \\ \text { tour } & \text { store tour option for participants. }\end{array}$

Follow-up Follow-up study visits and 24 hours ABPMs visit and 24 in late March 2020 were postponed until hours ABPM remote adaptations were made and approved by the IRB. Remote follow-up visits and 24 hours ABPMs were conducted using telephone calls and study materials mailed or dropped off at participants' houses. Weight and waist circumference measured using home scales and tape measures (rather than in clinic); clinic automated office blood pressure unable to be performed.

ABPM, ambulatory blood pressure monitoring; IRB, institutional review board.

able to continue the trial with adaptations although time windows were necessarily extended by approximately 1 month during March and April 2020 as there was a large degree of uncertainty on how to proceed and stay-athome orders in Pennsylvania were in effect.

\section{Sample size calculation}

In a meta-analysis of six trials comparing the effect of dietitian medical nutrition therapy to usual care, medical nutrition therapy reduced SBP by $4.7 \mathrm{~mm} \mathrm{Hg} .{ }^{26} \mathrm{In}$ the Atherosclerosis in Communities Study, every $1 \mathrm{~mm} \mathrm{Hg}$ population-wide decrease in SBP was associated with a reduction of 20.3 heart failure events, 12.1 stroke events and 13.5 coronary heart disease events in AfricanAmericans, and 13.3 heart failure events, 4.8 stroke events and 9.0 coronary heart disease events in whites.$^{27}$ Initially, the original sample size plan was for 160 patients, based on a conservative estimated drop-out rate of $25 \%$ and an SD of $8.8 \mathrm{~mm} \mathrm{Hg}$ change in 24-hour SBP to detect a difference of $4.6 \mathrm{~mm} \mathrm{Hg}$ between study arms. In order to determine whether there was a need to request additional funding from the funder (Geisinger Health Plan), a preplanned interim analysis was performed on $9 / 13 / 19$, showing that the SD for change in 24-hour SBP was larger 
than expected (SD 9.9) in the first 28 participants. Thus, a revised power calculation was performed. With an anticipated drop-out rate of $25 \%$, the updated sample size of 200 patients (150 completers) we expect to have $>80 \%$ power to detect a difference of $4.6 \mathrm{~mm} \mathrm{Hg}$ between study arms in change of 24-hour SBP. For other outcomes, we expect to have $>80 \%$ power to detect a difference of 6.9 in total HEI-2015 score with a SD of 15 units for change in total HEI-2015 score, and $>80 \%$ power to detect a difference of $2.1 \mathrm{~kg}$ between groups, assuming an SD of $4.5 \mathrm{~kg}$ for change in weight.

\section{Statistical analysis}

The primary contrast will be the between-group difference in 24-hour SBP from baseline to 12 weeks. The primary analyses will be intention to treat including all randomised participants who complete the trial with outcomes data, using unpaired t-tests. We will conduct complete case analyses; participants with missing data will be excluded from analyses. We will also examine whether 24-hour SBP improves at 12 weeks compared with baseline for each arm. A two-sided alpha of 0.05 will be used for analyses. Sensitivity analyses excluding non-adherent patients $(<25 \%$ days with complete dietary data) will also be conducted. In sensitivity analyses, we will compare the end of period values (not baseline change) with and without adjustment for baseline. We will test for an interaction between treatment assignment and baseline 24-hour SBP ( $\geq$ or $<130 \mathrm{mmHg}$ ) as an analysis of the DASH-Sodium trial found that low-sodium intake and the DASH diet had greater BP-lowering effects in those with higher baseline $\mathrm{SBP}^{28}$ Since it is possible that the effects of the interventions could be different during the COVID-19 pandemic, we will also conduct exploratory analyses testing whether change from baseline to 12 weeks in 24 hours SBP and other study outcomes differ in participants who completed the intervention prior to $3 / 1 / 2020$ versus participants who completed the intervention after 3/1/2020. All outcome measures will be assessed for a normal distribution, and non-normally distributed outcomes will be log-transformed. Likert scales will be assessed on a continuous scale or dichotomised. Continuous outcomes will be compared via linear regression, while dichotomised outcomes will be compared via logistic regression. Since the primary and secondary outcomes will be collected during study visits, no interim data on the outcomes will be available for patients who discontinue the study.

\section{Ethics and dissemination}

Initial ethical review and approval for this study was obtained from the Geisinger Institutional Review Board (IRB) on 10 January 2018 (current protocol version 1.22; approved 8/6/2020). Protocol amendments will be communicated to members of the study team and updated on the ClinicalTrials.gov website. Data will be collected using Research Electronic Data Capture REDCap) software on a secure, password-protected server. Study investigators are responsible for ensuring adherence to the protocol and guidelines for Good Clinical Practice. Symptomatic hypotension is possible in patients using BP-lowering medications and hypoglycaemic is possible for patients on diabetes medications undergoing dietary changes. Participants will be educated about the symptoms of hypotension and hypoglycaemic. Study staff will inform investigators of all patient adverse events occurring throughout the trial.

Dissemination of research will occur through several pathways. Research results will be submitted for publication to peer-reviewed journals and presented at national conferences. Summaries of Healthy BP findings will be provided locally to Geisinger leadership and healthcare providers and nationally to other stakeholders and through press releases. After completing the study, participants and their primary care providers receive a summary of study results.

\section{Patient and public involvement}

There was no specific patient or public involvement in the planning of this study. We will disseminate summary findings from this trial to study participants and to Geisinger's patient population.

\section{WHO TRIAL REGISTRATION DATA SET}

1. Primary registry and trial identifying number: ClinicalTrials.gov Identifier NCT03700710.

2. Date of registration in primary registry: 10/9/ 2018.

3. Secondary identifying numbers: N/A.

4. Source of monetary or material support: Geisinger health plan (grant number N/A).

5. Primary sponsor: Geisinger clinic.

6. Secondary sponsors: None.

7. Contact for public queries: Christina Yule; 570-2145233; cmyule@geisinger.edu.

8. Contact for scientific queries: Alex R. Chang, MD, MS; Kidney Health Research Institute; Geisinger; 100 n Academy Ave, Danville, PA; 570-271-8026; achang@ geisinger.edu.

9. Public title: Remote dietary counselling to promote healthy diet and BP.

10. Scientific title: Remote dietary counselling to promote healthy diet and BP.

11. Countries of recruitment: USA.

12. Health conditions studied: high BP.

13. Interventions:

Active Comparator: self-guided approach.

In the self-guided arm, participants will receive access to web-based tools to help achieve healthy lifestyle changes to lower their BP. The web-based tools include: (1) a web-based FFQ (Viocare FFQ), which will provide a snapshot of participants' dietary habits in the past 6 months as well as personalised recommendations for areas to improve; (2) access to BMIQ ( www.bmiq.com), an evidence-based programme developed by Louis Aronne at Columbia University, which 
includes programme materials for weight loss and leading a healthy lifestyle; (3) LoseIt (www.loseit.com), a meal-logging app that integrates seamlessly with the BMIQ website.

Experimental: dietitian-led approach

In the dietitian-led arm, dietitian will use motivational interviewing in 15-30 min telephone calls with participants. The BMIQ website will be used to share participant dietary data (LoseIt) and weight data with dietitians. Participants will receive access to web-based tools to help achieve healthy lifestyle changes to lower their BP. The web-based tools include: (1) a web-based FFQ (Viocare FFQ), which will provide a snapshot of participants' dietary habits in the past 6 months as well as personalised recommendations for areas to improve; (2) access to BMIQ; (3) LoseIt (www.loseit.com), a meal-logging app that integrates seamlessly with the BMIQ website.

14. Key inclusion and exclusion criteria

Inclusion Criteria: 24-hour ambulatory SBP 120-160 $\mathrm{mm} \mathrm{Hg}, \mathrm{BMI} \geq 25 \mathrm{~kg} / \mathrm{m}^{2}$, access to a telephone, access to a computer or smartphone with internet access, complete dietary data entry using LoseIt for at least 5 out of 7 days during run-in period, enter weight into the BMIQ portal during run-in period.

Exclusion Criteria: inability to understand English, myocardial infarction, stroke or atherosclerotic cardiovascular disease procedure within last 6 months, current treatment for malignancy, planned or previous bariatric surgery, pregnant, breast feeding or planned pregnancy prior to the end of participation, self-reported average consumption of $>21$ alcoholic beverages per week or binge drinking, psychiatric hospitalisation in past year, current symptoms of angina, planning to leave the area prior to end of the study, current participation in another clinical trial, principal investigator discretion (ie, concerns about safety, compliance).

15. Study type: randomised, parallel-arm, interventional study; study staff assessing outcomes and statistical analysis are masked. Purpose is to compare the efficacy of a self-guided versus a telehealth dietitian-led approach to lower BP through lifestyle modification.

16. Date of first enrolment-16 January 2019.

17. Sample size-200.

18. Recruitment status—recruiting.

19. Primary outcome-change in 24-hour systolic SBP (Time Frame: Baseline to 12-week follow-up) Measured by 24-hour ambulatory ABPM (SpaceLabs Ontrak).

20. Key secondary outcomes

Change in 24-hour diastolic DBP (Time Frame: Baseline to 12 weeks follow-up) Measured by 24-hour ambulatory ABPM (SpaceLabs Ontrak).

Change in Daytime Systolic SBP (Time Frame: Baseline to 12 week follow-up) Measured by 24-hour ambulatory ABPM (SpaceLabs Ontrak).
Change in Night-time Systolic SBP [ Time Frame: Baseline to 12 week follow-up ] Measured by 24-hour ambulatory ABPM (SpaceLabs Ontrak).

Change in Daytime DBP (Time Frame: Baseline to 12 week follow-up) Measured by 24-hour ambulatory MBPM (SpaceLabs Ontrak).

Change in Night-time Diastolic DBP (Time Frame: Baseline to 12 weeks follow-up) Measured by 24-hour ambulatory ABPM (SpaceLabs Ontrak).

Change in Total HEI - 2015 score (Time Frame: Baseline to 12 week follow-up) Assessed by Viocare FFQ (score $0-100,100=$ best possible score).

Change in Weight (Time Frame: Baseline to 12 week follow-up) Weight measured at baseline and 12 week visits using a calibrated scale without shoes.

Change in Waist Circumference (Time Frame: Baseline to 12 weeks follow-up) Measured using Gulick II tape measure.

Change in Physical Activity (MET-minute per week [ Time Frame: Baseline to 12 weeks follow-up]. Measured by IPAQ Short Form.

Change in Clinic SBP [ Time Frame: Baseline to 12 weeks follow-up ]Measured by average of 3 readings using Omron HEM 907XL.

Change in Clinic DBP [ Time Frame: Baseline to 12 weeks follow-up ] Measured by average of 3 readings using Omron HEM 907XL.

21. Ethics review-status approved, date of approval 2 October 2018.

22. Completion date-ongoing.

23. Summary results-N/A.

24. IPD sharing statement-Individual participant data collected for this trial will be made available to others; all available data will be deidentified. The protocol, statistical analysis plan, informed consent form and ethics committee approval will be made available.

Twitter Alex R Chang @alexchangmd

Acknowledgements A version of this work was presented at the American Heart Association EPI/Lifestyle 2020 meeting 3 March 2020 in Phoenix, Arizona. We thank Dr Louis Aronne and Guadalupe Minero for their guidance in setting up and customising the BMIQ online platform.

Contributors MT: writing original draft, review and editing; CY: project administration, investigation, review and editing; HB: project administration, investigation; SK: investigation, data curation, review and editing; CC: methodology, review and editing; AN: methodology, review and editing; SPJ: methodology, review and editing; LB-D: conceptualisation, funding acquisition, investigation, methodology, review and editing; ARC: conceptualisation, data curation, formal analysis, funding acquisition, investigation, methodology, supervision, review and editing. All authors have approved the final version.

Funding This work was supported by Geisinger Health Plan (award number N/A). ARC is supported by the National Institutes of Health/National Institute of Diabetes and Digestive and Kidney Diseases K23DK106515.

Disclaimer Funders had no role in the study design, collection, management, analysis, interpretation of data, or the decision to submit the report to publication.

Competing interests None declared.

Patient and public involvement Patients and/or the public were involved in the design, or conduct, or reporting, or dissemination plans of this research. Refer to the Methods section for further details.

Patient consent for publication Not required. 
Provenance and peer review Not commissioned; externally peer reviewed.

Open access This is an open access article distributed in accordance with the Creative Commons Attribution Non Commercial (CC BY-NC 4.0) license, which permits others to distribute, remix, adapt, build upon this work non-commercially, and license their derivative works on different terms, provided the original work is properly cited, appropriate credit is given, any changes made indicated, and the use is non-commercial. See: http://creativecommons.org/licenses/by-nc/4.0/.

\section{ORCID iDs}

Lisa Bailey-Davis http://orcid.org/0000-0002-8781-1521

Alex R Chang http://orcid.org/0000-0002-8114-7447

\section{REFERENCES}

1 Whelton PK, Carey RM, Aronow WS. 2017 ACC/AHA/AAPA ABC/ACPM/AGS/APhA/ASH/ASPC/NMA/PCNA guideline for the prevention, detection, evaluation, and management of high blood pressure in adults: a report of the American College of Cardiology/ American heart association Task force on clinical practice guidelines. Hypertension 2017.

2 Virani SS, Alonso A, Benjamin EJ, et al. Heart disease and stroke Statistics-2020 update: a report from the American heart association. Circulation 2020;141:e139-596.

3 Sacks FM, Svetkey LP, Vollmer WM, et al. Effects on blood pressure of reduced dietary sodium and the dietary approaches to stop hypertension (DASH) diet. DASH-Sodium Collaborative Research Group. N Engl J Med 2001;344:3-10.

4 Appel LJ, Champagne CM, Harsha DW, et al. Effects of comprehensive lifestyle modification on blood pressure control: main results of the premier clinical trial. JAMA 2003;289:2083-93.

5 Tuomilehto J, Jousilahti P, Rastenyte D, et al. Urinary sodium excretion and cardiovascular mortality in Finland: a prospective study. Lancet 2001;357:848-51.

6 Muntner P, Carey RM, Gidding S, et al. Potential U.S. population impact of the 2017 ACC/AHA high blood pressure guideline. J Am Coll Cardiol 2018;71:109-18.

7 Berman $\mathrm{AH}$, Kolaas K, Petersén E, et al. Clinician experiences of healthy lifestyle promotion and perceptions of digital interventions as complementary tools for lifestyle behavior change in primary care. BMC Fam Pract 2018;19:139.

8 Rubio-Valera M, Pons-Vigués M, Martínez-Andrés M, et al. Barriers and facilitators for the implementation of primary prevention and health promotion activities in primary care: a synthesis through metaethnography. PLoS One 2014;9:e89554.

9 Villinger $\mathrm{K}$, Wahl DR, Boeing $\mathrm{H}$, et al. The effectiveness of app-based mobile interventions on nutrition behaviours and nutrition-related health outcomes: a systematic review and meta-analysis. Obes Rev 2019;20:1465-84.

10 Appel LJ, Clark JM, Yeh H-C, et al. Comparative effectiveness of weight-loss interventions in clinical practice. $N$ Engl J Med 2011;365:1959-68.
11 Thomas JG, Bond DS, Raynor HA, et al. Comparison of smartphonebased behavioral obesity treatment with gold standard group treatment and control: a randomized trial. Obesity 2019;27:572-80.

12 Tucker KL, Sheppard JP, Stevens R, et al. Self-monitoring of blood pressure in hypertension: a systematic review and individual patient data meta-analysis. PLoS Med 2017;14:e1002389.

13 Omboni S, McManus RJ, Bosworth HB, et al. Evidence and recommendations on the use of telemedicine for the management of arterial hypertension: an international expert position paper. Hypertension 2020;76:1368-83.

14 Miller ER, Erlinger TP, Young DR, et al. Results of the diet, exercise, and weight loss intervention trial (DEW-IT). Hypertension 2002;40:612-8.

15 Chang AR, Bailey-Davis L, Hetherington V, et al. Remote dietary counseling using smartphone applications in patients with stages 13a chronic kidney disease: a mixed methods feasibility study. J Ren Nutr 2020;30:53-60.

16 Barenbaum SR, Mathews SE, Saunders KH, et al. Utility of BMIQ, a novel web-based weight management programme, at an academic weight management centre. Obes Sci Pract 2020;6:134-8.

17 Kristal AR, Kolar AS, Fisher JL, et al. Evaluation of web-based, selfadministered, graphical food frequency questionnaire. J Acad Nutr Diet 2014:114:613-21.

18 Carver C, Scheier M. On the self regulation of behavior. New York: Cambridge University Press, 1998.

19 Lave J, Wenger E. Situated learning: legitimate peripheral participation. New York: Cambridge University Press, 1991.

20 Baena Díez JM, Carrera Morodo M, Corral Roca M. Impact of the new criteria of the ACC/AHA on the diagnostic prevalence of hypertension. Med Clin 2020;154:254-6.

21 de Greeff A, Shennan AH. Validation of the Spacelabs 90227 OnTrak device according to the European and British hypertension societies as well as the American protocols. Blood Press Monit 2020:25:110-4.

22 Craig CL, Marshall AL, Sjöström M, et al. International physical activity questionnaire: 12 -country reliability and validity. Med Sci Sports Exerc 2003;35:1381-95.

23 Reedy J, Lerman JL, Krebs-Smith SM, et al. Evaluation of the healthy eating Index-2015. J Acad Nutr Diet 2018;118:1622-33.

24 Griebeler ML, Levis S, Beringer LM, et al. Self-reported versus measured height and weight in Hispanic and non-Hispanic menopausal women. J Womens Health 2011;20:599-604.

25 Carranza Leon BG, Jensen MD, Hartman JJ, et al. Self-Measured vs Professionally measured waist circumference. Ann Fam Med 2016;14:262-6.

26 Sikand G, Cole RE, Handu D, et al. Clinical and cost benefits of medical nutrition therapy by registered dietitian nutritionists for management of dyslipidemia: a systematic review and meta-analysis. $J$ Clin Lipidol 2018;12:1113-22.

27 Hardy ST, Loehr LR, Butler KR, et al. Reducing the blood pressurerelated burden of cardiovascular disease: impact of achievable improvements in blood pressure prevention and control. J Am Heart Assoc 2015;4:e002276.

28 Juraschek SP, Miller ER, Weaver CM, et al. Effects of sodium reduction and the DASH diet in relation to baseline blood pressure. $J$ Am Coll Cardiol 2017;70:2841-8. 